Keywords: Tritium

Polymers

Radiation Damage

Electrically conductive polymers

Retention - Permanent

\title{
Effects of Tritium Gas Exposure on Electrically Conducting Polymers
}

\author{
Marie C. Kane, Elliot A. Clark, and Robert J. Lascola
}

Publication Date: December 2009

Savannah River National Laboratory

Savannah River Nuclear Solutions

Aiken. SC 29808 


\section{DISCLAIMER}

This work was prepared under an agreement with and funded by the U.S. Government. Neither the U. S. Government or its employees, nor any of its contractors, subcontractors or their employees, makes any express or implied:

1. warranty or assumes any legal liability for the accuracy, completeness, or for the use or results of such use of any information, product, or process disclosed; or

2. representation that such use or results of such use would not infringe privately owned rights; or 3. endorsement or recommendation of any specifically identified commercial product, process, or service. Any views and opinions of authors expressed in this work do not necessarily state or reflect those of the United States Government, or its contractors, or subcontractors.

Printed in the United States of America

Prepared for

U.S. Department of Energy 
Executive Summary ............................................................................................................................1

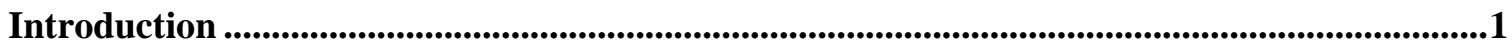

Experimental Program ....................................................................................................................2

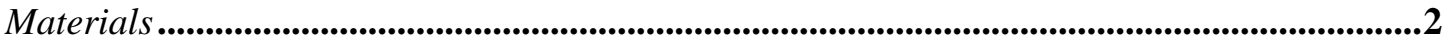

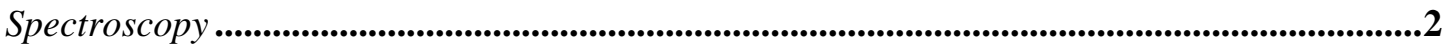

Beta radiation (tritium) exposures................................................................................................................3

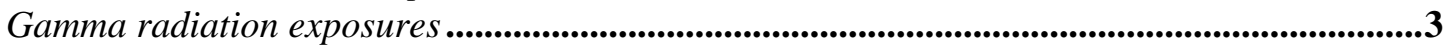

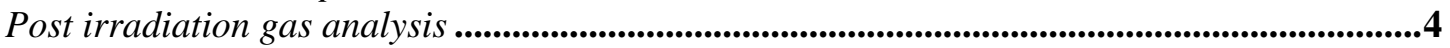

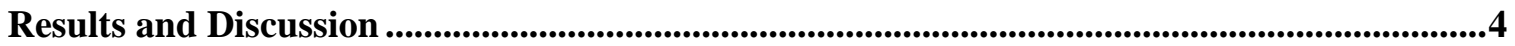

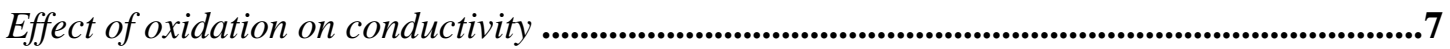

Raman spectroscopy of irradiated PEDOT and PANi samples...............................................8

UV-Visible spectroscopy of irradiated PANi and PEDOT-PSS samples...................................9

Discussion on spectroscopy results ..................................................................................................11

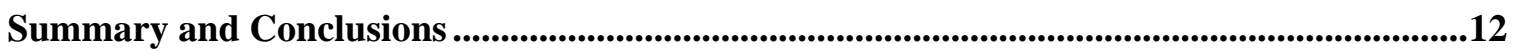

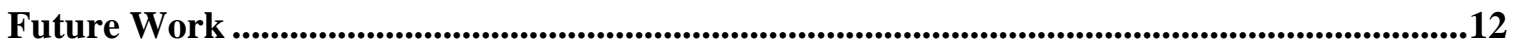

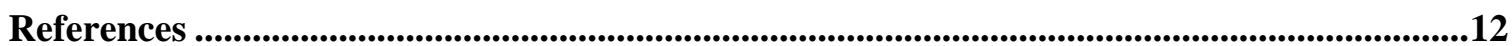




\section{TABLES}

1. Tritium exposure experiments with test number, exposure time, and exposure pressure ...........

2. Gamma radiation exposure experiments with test number, total dose, and exposure environment.

3. Dose dependence of peak absorbance of PANi polaron band

FIGURES

Page

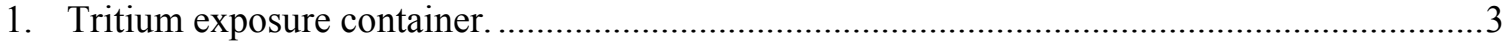

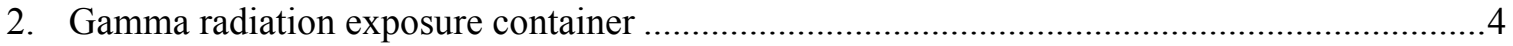

3. Data for tritium exposures at $1 \mathrm{~atm}$ pressure tritium gas...................................................

4. Data for tritium exposures at $0.1 \mathrm{~atm}$ pressure tritium gas. ................................................6

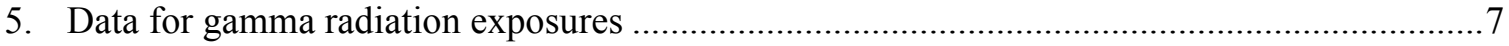

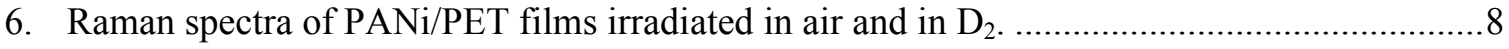

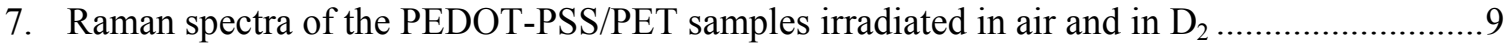

8. Absorption spectra of the air-irradiated and $\mathrm{D}_{2}$-irradiated PANi samples.............................10 


\section{EXECUTIVE SUMMARY}

Effects of beta (tritium) and gamma irradiation on the surface electrical conductivity of two types of conducting polymer films are documented to determine their potential use as a sensing and surveillance device for the tritium facility. It was shown that surface conductivity was significantly reduced by irradiation with both gamma and tritium gas. In order to compare the results from the two radiation sources, an approximate dose equivalence was calculated. The materials were also sensitive to small radiation doses $\left(<10^{5} \mathrm{rad}\right)$, showing that there is a measurable response to relatively small total doses of tritium gas. Spectroscopy was also used to confirm the mechanism by which this sensing device would operate in order to calibrate this sensor for potential use. It was determined that one material (polyaniline) was very sensitive to oxidation while the other material (PEDOT-PSS) was not. However, polyaniline provided the best response as a sensing material, and it is suggested that an oxygen-impermeable, radiation-transparent coating be applied to this material for future device prototype fabrication.

\section{INTRODUCTION}

A great deal of interest has developed in recent years in the area of conducting polymers due to the high levels of conductivity that can be achieved, some comparable to that of metals [Gerard 2002]. Additionally, the desirable physical and chemical properties of a polymer are retained and can be exploited for various applications, including light emitting diodes (LED), anti-static packaging, electronic coatings, and sensors. The electron transfer mechanism is generally accepted as one of electron "hopping" through delocalized electrons in the conjugated backbone, although other mechanisms have been proposed based on the type of polymer and dopant [Inzelt 2000, Gerard 2002]. The conducting polymer polyaniline (PANi) is of particular interest because there are extensive studies on the modulation of the conductivity by changing either the oxidation state of the main backbone chain, or by protonation of the imine groups [de Acevedo, 1999].

There are several types of radiation sensors commercially available, including ionization chambers, geiger counters, proportional counters, scintillators and solid state detectors. Each type has advantages, although many of these sensors require expensive electronics for signal amplification, are large and bulky, have limited battery life or require expensive materials for fabrication. A radiation sensor constructed of a polymeric material could be flexible, light, and the geometry designed to suit the application. Very simple and inexpensive electronics would be necessary to measure the change in conductivity with exposure to radiation and provide an alarm system when a set change of conductivity occurs in the sensor that corresponds to a predetermined radiation dose having been absorbed by the polymer. The advantages of using a polymeric sensor of this type rather than those currently in use are the flexibility of sensor geometry and relatively low cost. It is anticipated that these sensors can be made small enough for glovebox applications or have the ability to monitor the air tritium levels in places where a traditional monitor cannot be placed.

There have been a few studies on the changes in conductivity of polyaniline specifically for radiation detection [de Acevedo, 1999; Lima Pacheco, 2003], but there have been no reports on the effects of tritium (beta radiation) on conducting polymers, such as polyaniline or polythiophene. The direct implementation of conducting polymers as radiation sensor materials has not yet been commercialized due to differing responses with total dose, dose rate, etc. Some have reported a large increase in the surface conductivity 
with radiation dose while others report a marked decrease in conductive properties; these differing observations may reflect the competing mechanisms of chain scission and cross-linking. However, it is clear that the radiation dose effects on conducting polymers must be fully understood before these materials can be used as sensing devices.

This report presents the results of irradiations of two conductive polymers: polyaniline and polythiophene. Samples of doped polyaniline and polythiophene were coated onto polyester (polyethyleneterephalate, PET) substrates and were exposed to both tritium gas (beta irradiation) and ${ }^{60} \mathrm{Co}$ gamma irradiation. The samples were subsequently characterized after various total doses. Infrared spectroscopy was utilized to characterize the gamma-exposed samples post-irradiation. Although the sources of radiation are different in kind (charged particle versus photon) and their energies differ, there will be great value in using noncontaminating gamma irradiation to model the effects of tritium beta radiation.

It is hoped that these results can be exploited in the future to fabricate a prototype sensor for use in the tritium facility for short and long term sensing and surveillance applications where an alternative sensor device is needed.

This task is incorporated in the SRS Enhanced Surveillance Campaign (ESC) as Task TSR 15 "Effect of Tritium Exposure on Reservoir Polymer Materials", and this report fulfills Savannah River Site Level III milestone "Issue Summary Report on Tritium Reservoir Materials Aging Studies" and supports the Enhanced Surveillance Campaign Level II milestone 3145, "Complete an annual Enhanced Surveillance stockpile aging assessment report to support the annual assessment process".

\section{EXPERIMENTAL PROGRAM}

\section{Materials}

Samples of polyaniline doped with dinonylnaphthalenesulfonic acid (PANi-DSS) and poly(3,4ethylenedioxythiophene)-poly(styrenesulfonate) (PEDOT-PSS) polymers were obtained from Crosslink, USA. The trade names for these materials are APC 4147 and APC $8116 \mathrm{D}$, respectively. The polymers were solution-cast as coatings approximately $25 \mu \mathrm{m}$ thick on a PET substrate for mechanical stability. The polyaniline polymer is the conducting form- emeraldine salt. PET was chosen for its relative resistance to radiation degradation due to the presence of aromatic groups [Clough and Shalaby, 1996]. The samples were supplied as large sheets that were cut into strips for radiation exposure.

\section{Spectroscopy}

Raman spectra of the polymer films were taken with a VERAX spectrometer (InPhotonics, Norwood, MA), using $80 \mathrm{~mW}$ of laser power and an excitation wavelength of $785 \mathrm{~nm}$. Higher laser powers caused physical damage to the PANi films. Spectra were taken in the backscattering geometry $\left(180^{\circ}\right)$ using a fiber probe with a $5 \mathrm{~mm}$ focal length. Films were cut and stacked in triplicate to increase the Raman signal. No fluorescence was observed for any of the samples. Spectra were obtained for Raman shift frequencies of 200-1800 $\mathrm{cm}^{-1}$. Spectral processing was performed using GRAMS (Thermo Fisher Scientific, Waltham, MA).

Absorbance spectra were obtained over the range 250-1100 nm using a TIDAS II diode array spectrometer (J\&M, Essingen, Germany), with a combined deuterium/tungsten-halogen light source. Spectra were obtained for single thicknesses of the film. Spectra were taken using air as a reference. The instrument was calibrated against reference emission lines from an $\mathrm{Hg} / \mathrm{Ar}$ lamp. 


\section{Beta radiation (tritium) exposures}

The polymer (PANi-DSS and PEDOT-PSS coated PET) samples were exposed to tritium gas (beta radiation) in stainless steel exposure containers specifically designed for this purpose (Figure 1). Each container had a volume of $55 \mathrm{~cm}^{3}$, and $3-6$ samples were placed in each container. The containers were then attached to a tritium gas manifold and loaded with either 760 Torr or 76 Torr pressure of tritium gas. The containers were then sealed by two valves, and the polymer samples were exposed to tritium for various lengths of time, according to the schedule listed in Table 1. The equivalent dose of tritium (in rad) absorbed by the samples was calculated by assuming a tritium concentration in the polymer samples of $10 \mathrm{Ci} / \mathrm{g}$ at 760 Torr and $1 \mathrm{Ci} / \mathrm{g}$ at 76 Torr. These concentration estimates are based on the solubility of tritium gas in the samples measured for other materials completed at the Savannah River Site [Clark, 2008].

After the prescribed exposure time, the total pressure and composition of the gas in the container were characterized using GC mass spectrometry. A surface conductivity meter (Prostat, model PRS 812) with small sample concentric ring probe was used to measure the surface conductivity of the samples in a tritium hood (open-air environment, denoted as "in Air" in Figures 3 and 4) and also in a nitrogen glove box (oxygen-free environment, denoted as "in N2" in Figures 3 and 4) before and after exposure. The two different measurements (air and nitrogen environments) were used to examine the effects of moisture and oxygen on the conductivity measurement itself. Unexposed control samples were characterized using the same procedures and facilities as exposed samples.

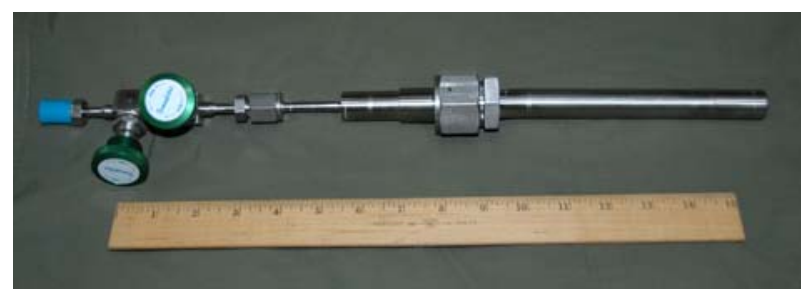

Figure 1. Tritium exposure container.

Table 1. Tritium exposure experiments with test number, exposure time, and exposure pressure.

\begin{tabular}{|c|c|c|c|c|}
\hline $\begin{array}{c}\text { Experiment } \\
\text { number }\end{array}$ & $\begin{array}{c}\text { Exposure } \\
\text { Time (hour) }\end{array}$ & $\begin{array}{c}\text { Exposure } \\
\text { Pressure (torr) }\end{array}$ & $\begin{array}{c}\text { Equivalent } \\
\text { Dose (rad) }\end{array}$ & $\begin{array}{c}\text { Approximate } \\
\text { dose (rad) }\end{array}$ \\
\hline 1 & 0.1 & 76 & 1213 & $10^{3}$ \\
\hline 2 & 0.1 & 760 & 12130 & $10^{4}$ \\
\hline 3 & 1 & 76 & 12130 & $10^{4}$ \\
\hline 4 & 1 & 760 & 121300 & $10^{5}$ \\
\hline 5 & 10 & 76 & 121300 & $10^{5}$ \\
\hline 6 & 10 & 760 & 1213000 & $10^{6}$ \\
\hline 7 & 100 & 76 & 1213000 & $10^{6}$ \\
\hline 8 & 100 & 760 & 12130000 & $10^{7}$ \\
\hline 9 & 1000 & 76 & 12130000 & $10^{7}$ \\
\hline 10 & 1000 & 760 & 121300000 & $10^{8}$ \\
\hline
\end{tabular}

\section{Gamma radiation exposures}

The PANi-DSS and PEDOT-PSS samples were exposed to gamma radiation in stainless steel exposure containers specifically designed for this purpose (Figure 2). The wall thickness of the container was chosen to minimize attenuation of the gamma radiation; the attenuation was considered negligible. $\mathrm{A}{ }^{60} \mathrm{Co}$ source was used with a dose rate of $3.5 \times 10^{5} \mathrm{rad} / \mathrm{hr}(3.5 \mathrm{kGy} / \mathrm{hr})$ with a total dose for each sample according to the 
experimental design in Table 2. Once the samples were placed into the container, it was filled with either air or deuterium gas at $1 \mathrm{~atm}$ pressure. The exposures were completed in both environments to examine the effect of oxygen present during irradiation. The surface conductivity of the samples was measured in the same manner as the beta irradiation samples.

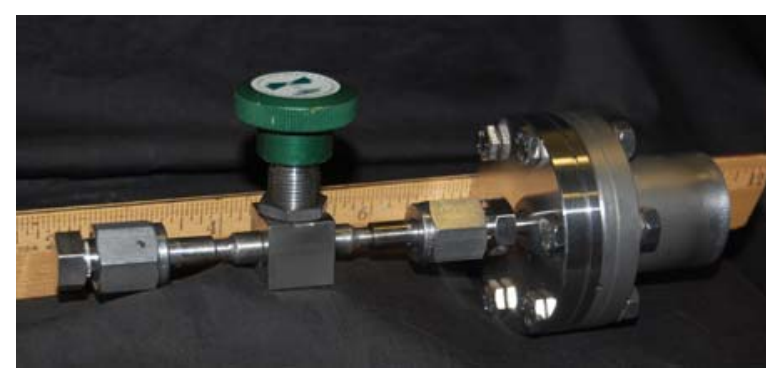

Figure 2. Gamma radiation exposure container.

Table 2. Gamma radiation exposure experiments with test number, total dose, and exposure environment.

\begin{tabular}{|c|c|c|}
\hline $\begin{array}{c}\text { Experiment } \\
\text { number }\end{array}$ & $\begin{array}{c}\text { Total dose } \\
(\mathrm{rad})\end{array}$ & $\begin{array}{c}\text { Exposure } \\
\text { environment }\end{array}$ \\
\hline 1 & $10^{5}$ & air \\
\hline 2 & $10^{5}$ & deuterium \\
\hline 3 & $10^{6}$ & air \\
\hline 4 & $10^{6}$ & deuterium \\
\hline 5 & $10^{7}$ & air \\
\hline 6 & $10^{7}$ & deuterium \\
\hline 7 & $10^{8}$ & air \\
\hline 8 & $10^{8}$ & deuterium \\
\hline 9 & $10^{9}$ & air \\
\hline 10 & $10^{9}$ & deuterium \\
\hline
\end{tabular}

\section{Post Irradiation Gas Analysis}

The post irradiation gas analysis was performed on an Agilent Model M200 Micro Gas Chromatograph with a 5A 10-meter molecular sieve column. The column head pressure was $25 \mathrm{psig}$. The presence of hydrogen in the gas samples was determined by first calibrating to a known concentration of hydrogen gas in deuterium.

\section{RESULTS AND DISCUSSION}

Tritium exposure (beta irradiation) data at two different pressures (dose rates) and gamma irradiation data were plotted with respect to measured surface conductivity at particular total doses (Figures 3, 4 and 5). Overall, there was a marked decrease in surface conductivity at the highest total dose for all of the samples exposed to tritium and gamma radiation. For this analysis, it is assumed that there are two main competing mechanisms for the change in electrical properties of PANi and PEDOT-PSS when in the presence of an ionizing radiation field. The first mechanism is free radical formation along the polymer backbone via chain scission. It is assumed that when free radical formation is the dominant process during irradiation at a particular total dose, the surface conductivity would decrease due to defects being formed in the electrical pathway through the sample. At relatively low total doses, free radical formation dominates the kinetics of polymer degradation, and the relative surface conductivity was shown to decrease in all but one sample from the original measured conductivity. 
Once these free radicals are formed, several subsequent reactions can take place. The free radical may react with other free radicals, for example recombining or cross-linking, to essentially reform the electrical pathway through the sample. In this case, the relative surface conductivity would theoretically increase. If the cross-link density becomes higher than that of the original polymer before irradiation, the surface conductivity may increase above that of the starting material, as is the case of PEDOT-PSS exposed in a tritium atmosphere. The free radical may also react with oxygen, if present (see below), for a reduction in surface conductivity due to the prevention of further cross-linking. It is assumed that both the mechanisms of chain scission and cross-linking are occurring randomly and simultaneously, so it is impossible to discern between the two. The predominance of one mechanism (e.g. chain scission) over the other (e.g. crosslinking) dictates the increase or decrease of measured surface conductivity with total dose.

For the tritium exposures, it can be concluded that $10^{7}$ rad equivalent total dose is the point at which extensive chain scission occurs in greater number than chain recombination (cross-linking) in both polymers and the surface conductivity is reduced by several orders of magnitude. The PEDOT-PSS samples can experience both chain scission and cross-linking as the dominant mechanism at different total doses, while PANi is dominated by chain scission at almost every recorded total dose. This trend also holds true for the gamma irradiated samples.

Two different pressures of tritium gas were used to simulate two dose rates of beta radiation. It is clear that the lower dose rate caused much more severe chain scission (reduction in conductivity) than the higher dose rate. This dose rate effect seen even in vacuum has been confirmed by others [Biskman, 2004]

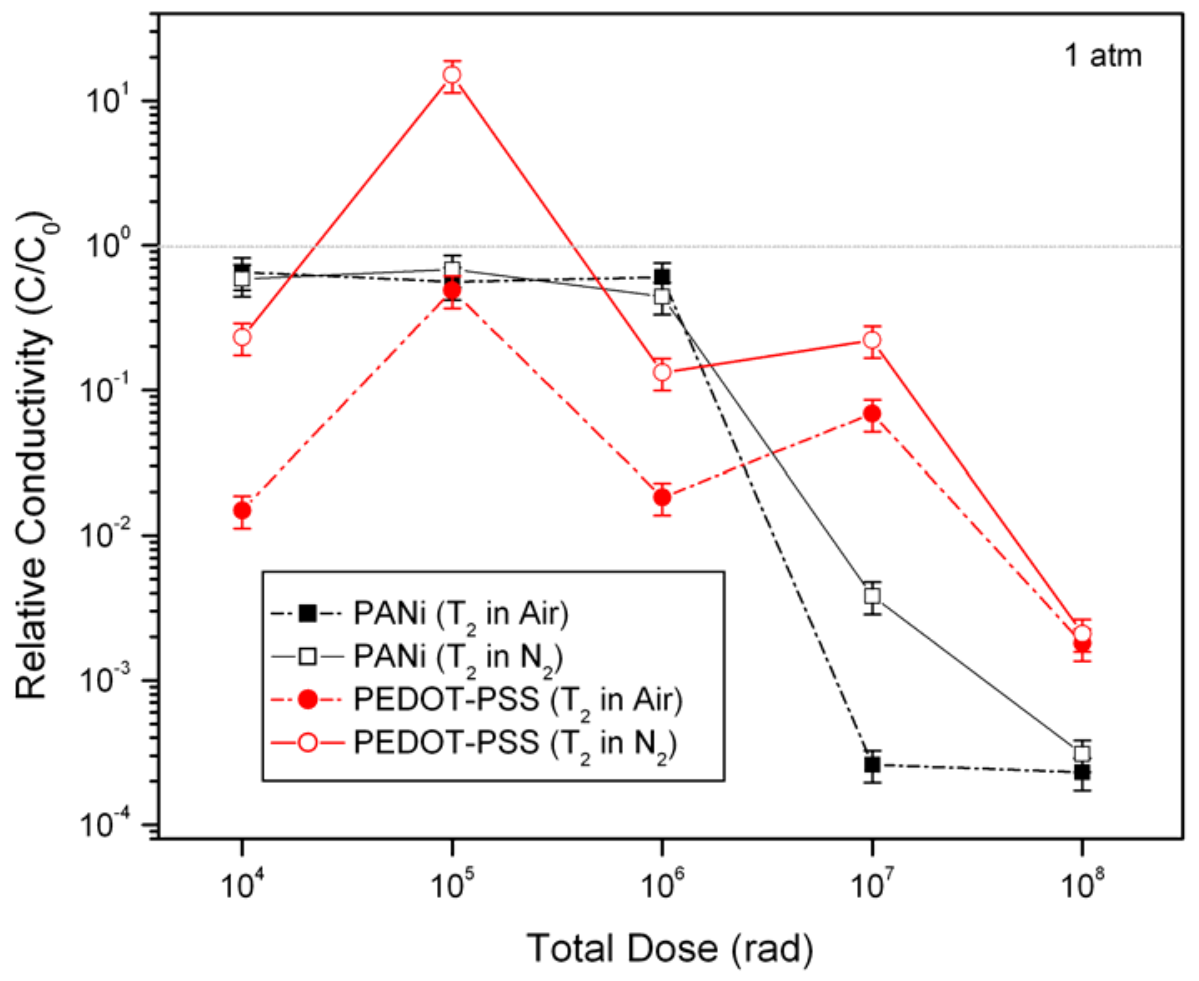

Figure 3. Data for tritium exposures at 1 atm pressure tritium gas. 


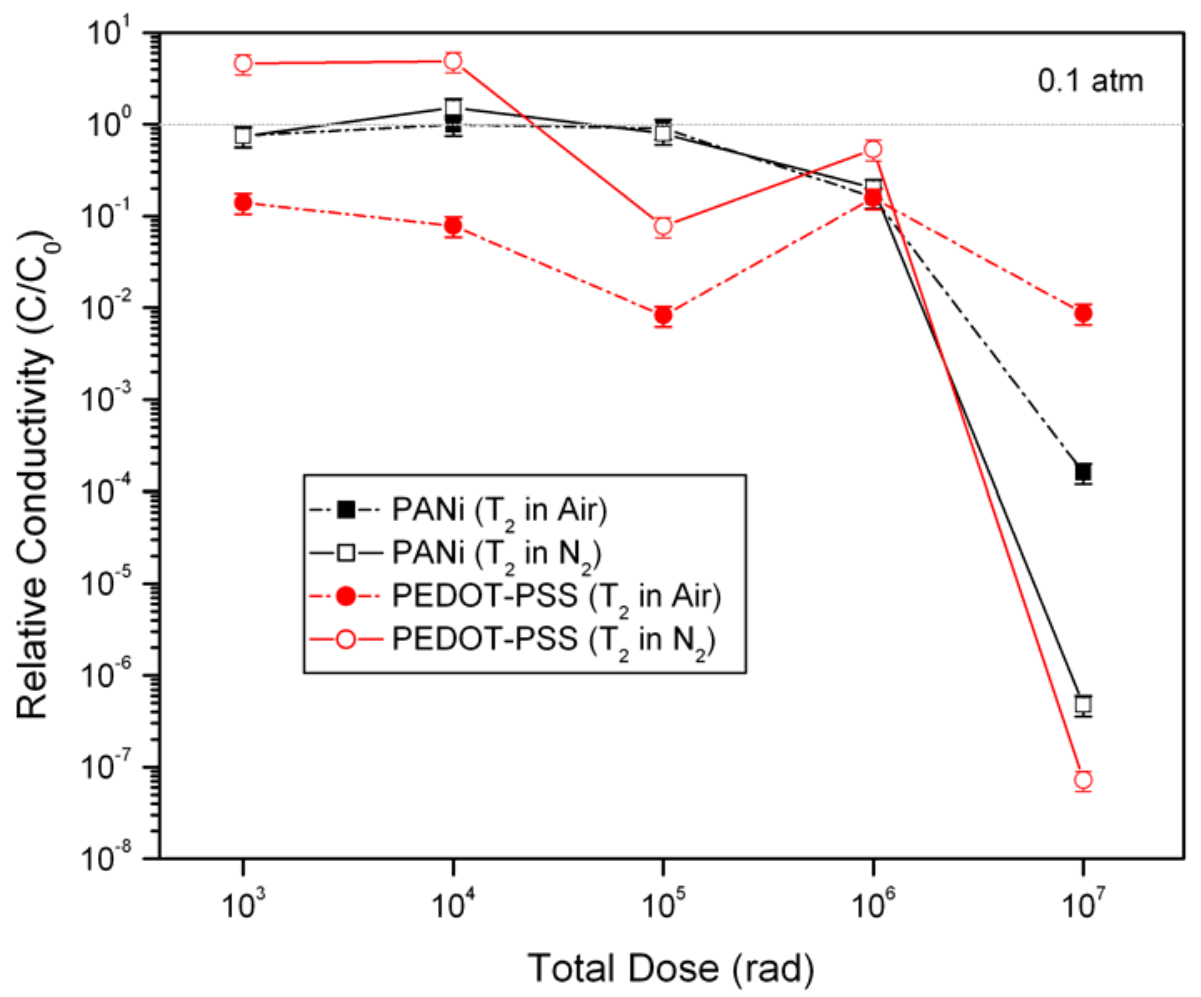

Figure 4. Data for tritium exposures at $0.1 \mathrm{~atm}$ pressure tritium gas. 


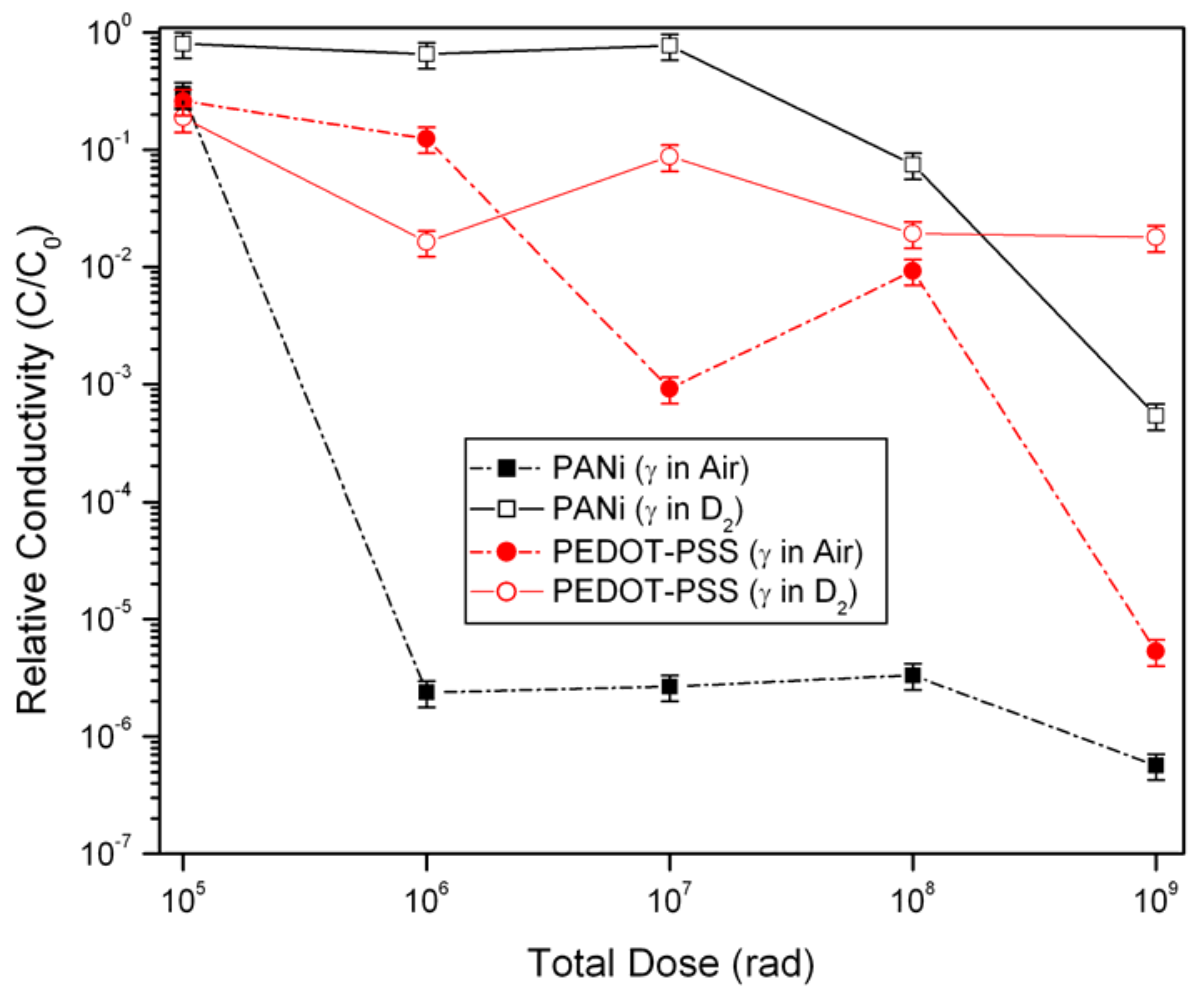

Figure 5. Data for gamma radiation exposures.

\section{Effect of oxidation on conductivity}

The presence of oxygen (air) during gamma irradiation affected the conductivity greatly as compared to samples exposed in a non-air environment (Figure 5). Polyaniline was much more affected by oxidation effects than PEDOT-PSS with a drop in conductivity of over five orders of magnitude. Upon initial irradiation, free radicals are formed within the polymer and readily react with free oxygen $\left(\mathrm{O}_{2}\right)$ according to the following reaction to form peroxy radicals

$$
\mathrm{R}^{\bullet}+\mathrm{O}_{2} \rightarrow \mathrm{ROO}^{\bullet}
$$

The participation of oxygen during the beginning of this free-radical chain reaction often leads to increased chain scission activity [Clough, 1994]. The formation of these peroxy radicals prevents further cross-linking. This reduction in conductivity between samples exposed in air or deuterium can be clearly seen in the gamma irradiation samples (Figure 5), and most prevalently for PANi. The larger decrease in conductivity for polyaniline can most likely be attributed to the greater concentration of double bonds than that of PEDOT-PSS. Double bonds are particularly susceptible to degradation (attack) by ionized oxygen species (e.g. ozone) [Clegg, 1991]. Oxidation degradation is also limited by oxygen diffusion kinetics, or how quickly the radicals can migrate to the oxidation surface. Both of these mechanisms are slowed by increased crystallinity in the polymer due to diffusion taking place only in the amorphous regions. Typically, PANi is more crystalline than PEDOT-PSS due to less bulky pendant groups. 
In the case of the tritium exposures, no oxygen was present in the exposure vessels and conductivity decrease due to oxidation only took place during the time that the samples were taken out of the exposure vessel and moved into an air (oxygen) environment for testing. This process took no longer than 30 minutes. In this case, the PEDOT-PSS was more susceptible to environmental issues (oxygen, moisture) than the PANi, as compared to the samples measured in $\mathrm{N}_{2}$. It may be concluded from these results that oxidation occurs on a long time scale for PANi and a short time scale for PEDOT-PSS, or that there is a greater concentration of free radicals present on the surface after irradiation on the PEDOT-PSS sample than PANi, which can participate in the oxidation mechanism.

\section{Raman spectroscopy of irradiated PANi and PEDOT-PSS samples}

The Raman spectra of PANi/PET films irradiated in air and in $\mathrm{D}_{2}$ are shown in Figure 6. Spectra are offset for clarity, and normalized to the PET peak at $1605 \mathrm{~cm}^{-1}$. Peaks corresponding to PET vibrations were identified by comparison to spectra of the uncoated PET substrate and confirmed by comparison to literature reports [Michielsen, 2001] Peaks due to the PANi layer (identified by "A") also match well with literature spectra of the emeraldine salt structure [Kim, 2007]. The spectral similarity to emeraldine salt is expected, as the $785 \mathrm{~nm}$ excitation wavelength provides resonance enhancement with the $\pi-\pi^{*}$ near-infrared (NIR) absorption band of that structure.
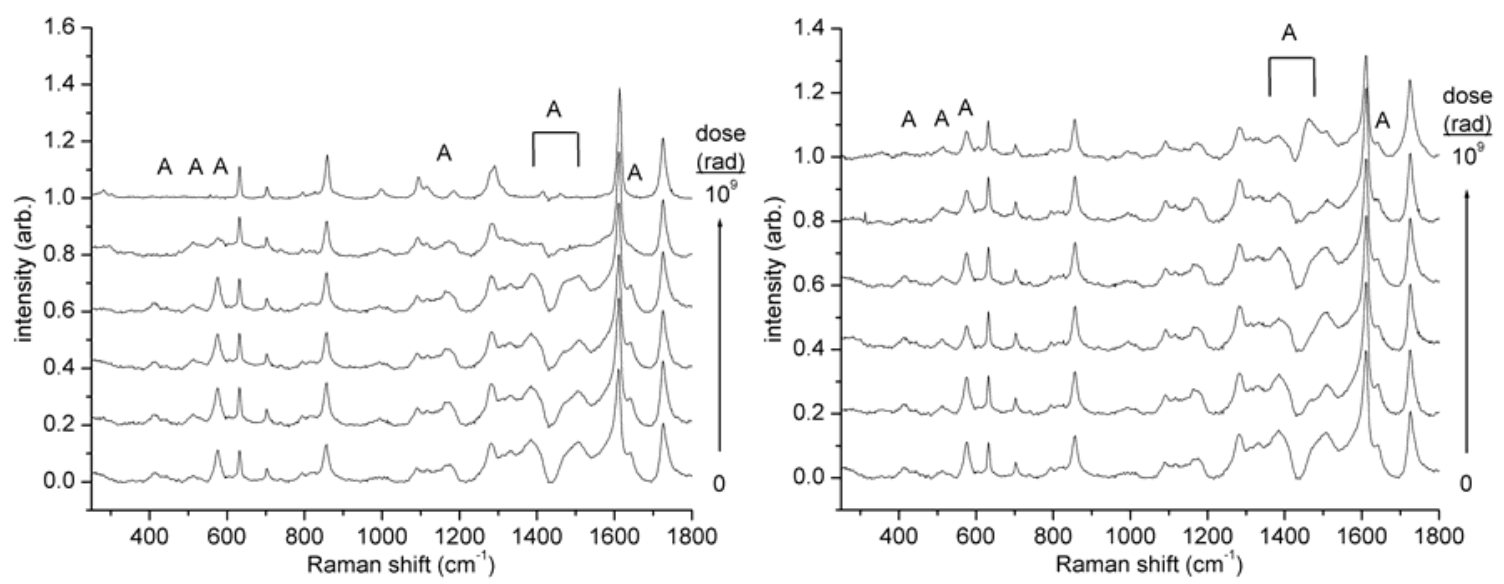

Figure 6. Raman spectra of PANi/PET films irradiated in air (left) and in $\mathrm{D}_{2}$ (right).

The dependence of the Raman spectra on radiation dose differs for the two atmospheres. The spectra of the PANi/PET samples irradiated in air are similar for irradiation doses up to $10^{7} \mathrm{rad}$. At $10^{8} \mathrm{rad}$, the PANi peaks are significantly diminished relative to the PET peaks. At $10^{9} \mathrm{rad}$, the PANi peaks have completely disappeared, and the spectrum is equivalent to that of the plain, unirradiated PET film, despite the yellowing and brittleness of the material. There are no isolated peaks that appear or grow with neither increasing dose, nor do the existing line shapes change substantially. All the PANi peaks in the $10^{8}$ rad sample are diminished equally relative to the PET peaks.

In contrast to the air-irradiated samples, the samples irradiated in $\mathrm{D}_{2}$ do not show any substantial spectral changes for any dose, including $10^{9} \mathrm{rad}$. Note that the range of the Raman spectra precludes observation of any $\mathrm{C}-\mathrm{H}$ peaks, which might be sensitive to changes in the acid/base form of the PANi, nor of any C-D peaks, which would indicate hydrogen isotopic exchange.

The Raman spectra of the PEDOT-PSS/PET samples irradiated in air and in $\mathrm{D}_{2}$ are shown in Figure 7. Peaks corresponding to PEDOT-PSS are identified by "D". The PET peaks have the same frequencies as those observed in the PANi samples. The PEDOT-PSS peak positions are consistent with those observed in the spectrum reported by Garreau et al. [Garreau, 1999] for an electrochemically prepared sample excited at 
$1064 \mathrm{~nm}$. Since neither 785 nor $1064 \mathrm{~nm}$ excitation will give rise to resonance enhancement, the Raman spectra for these wavelengths should be similar. A series of overlapping peaks in the region of 1350-1550 $\mathrm{cm}^{-1}$ has been assigned as being associated with $\mathrm{C}-\mathrm{C}\left(\sim 1370 \mathrm{~cm}^{-1}\right)$ and $\mathrm{C}=\mathrm{C}\left(\sim 1430 \mathrm{~cm}^{-1}\right)$ stretches. Small peaks found below $600 \mathrm{~cm}^{-1}$ are due to ring deformation modes.
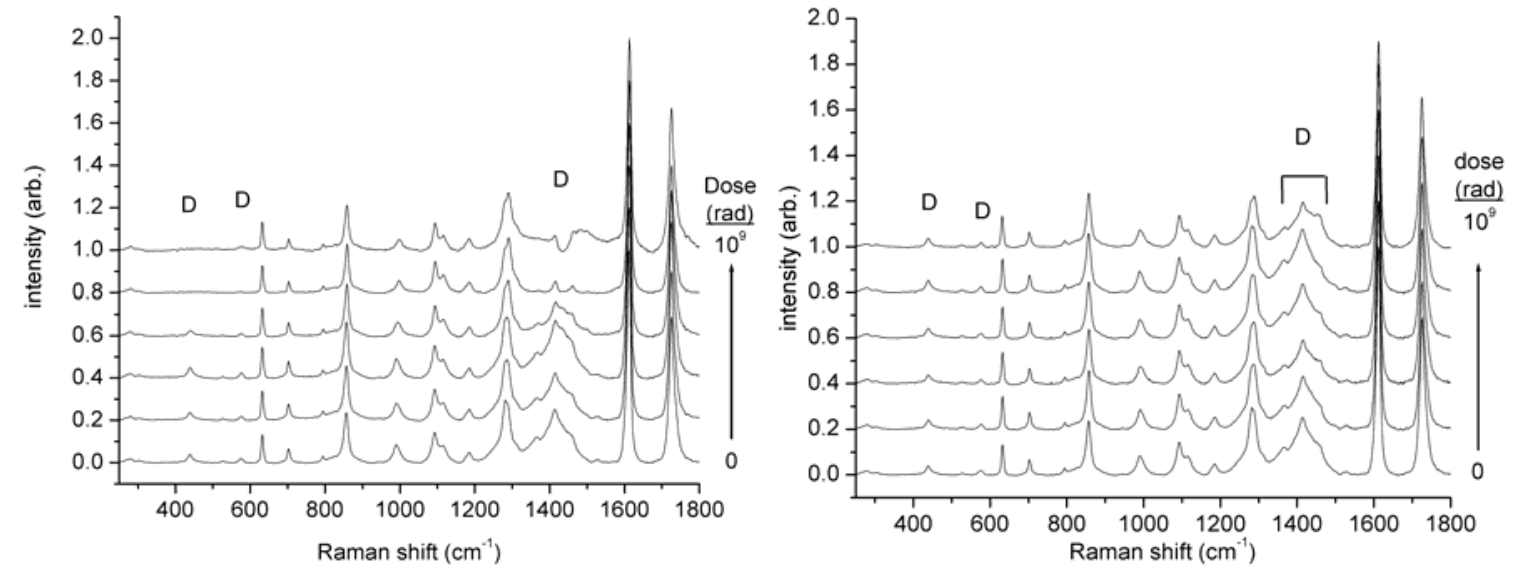

Figure 7. Raman spectra of the PEDOT-PSS/PET samples irradiated in air (left) and in $\mathrm{D}_{2}$ (right).

The general trend of changes in the Raman spectrum of the PEDOT-PSS films with increasing dose is similar to that observed for the PANi. PEDOT-PSS is seen to be destroyed with sufficient irradiation in air, while it is apparently unaffected (up to $10^{9} \mathrm{rad}$ ) in $\mathrm{D}_{2}$. The decrease of the PEDOT-PSS peaks is first observed at $10^{7}$ $\mathrm{rad}$, with complete loss at $10^{8} \mathrm{rad}$, in contrast to the $10^{8} / 10^{9} \mathrm{rad}$ threshold for PANi. As was observed for the PANi samples, no new peaks appeared with higher doses.

The shape of overlapped PEDOT-PSS C-C stretching bands changes more for the air samples than the $\mathrm{D}_{2}$ samples. This observation could indicate that the ratio of carbon single to double bonds is changing.

However, interpretation of these changes must take into account the presence of two PET peaks at $\sim 1415$ and $\sim 1460 \mathrm{~cm}^{-1}$. A decrease of PEDOT-PSS relative to PET in the sample will also change the spectrum in the direction of the observed change. Attempts to perform least-squares fits of the spectra to a combination of the two PEDOT-PSS and two PET peaks were inconclusive, as the uncertainty in fit parameters over multiple measurements exceeded any dose-related trends of those parameters. Future analyses with higherquality data may allow the observation of any conformational changes.

\section{UV-Visible spectroscopy of irradiated PANi and PEDOT-PSS samples}

\section{Absorption spectroscopy of irradiated PANi samples}

The absorption spectra of the air-irradiated PANi samples are shown in Figure 8. The spectra display previously reported absorption features of the emeraldine salt form, including bands at 445 and $\sim 800 \mathrm{~nm}$, which are related to the $\pi_{\mathrm{B}}-\pi_{\mathrm{S}}$ polaron band [Chan, 2002]. There is no evidence of absorptions at 330 and $590 \mathrm{~nm}$, which would arise from the emeraldine base. Shorter wavelength absorbances can be attributed to the PET substrate. 

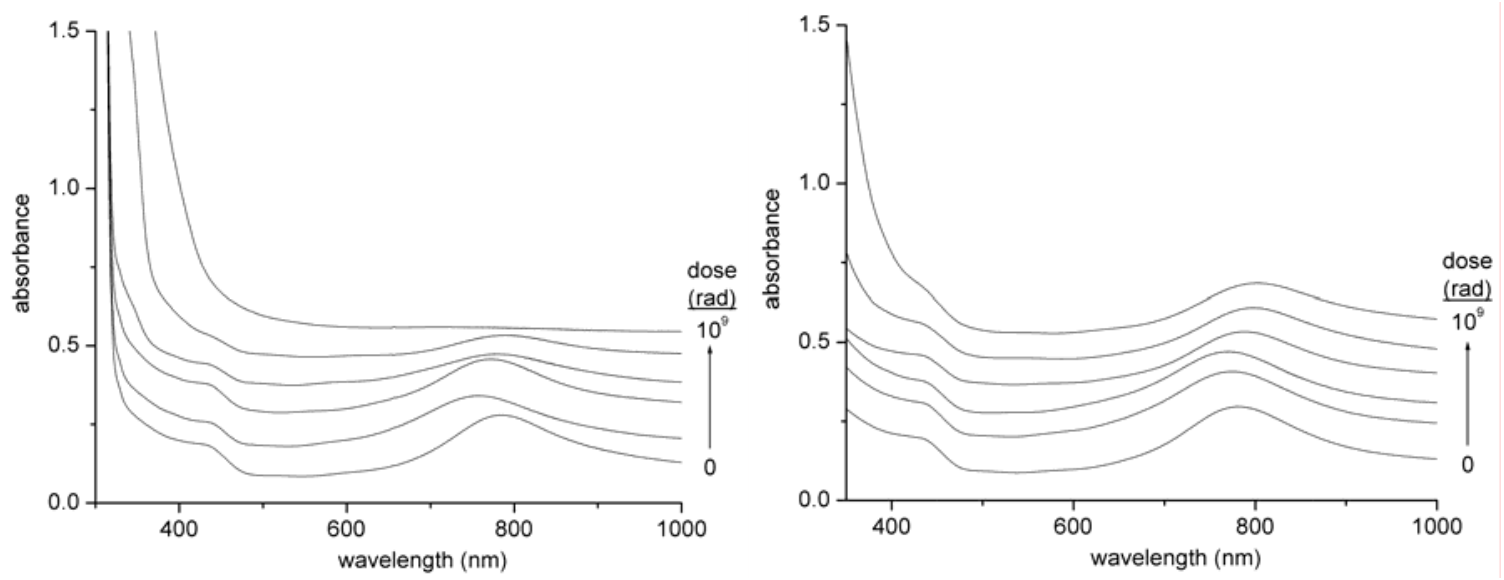

Figure 8. Absorption spectra of the air-irradiated (left) and $\mathrm{D}_{2}$-irradiated (right) PANi samples.

The position of maximum absorbance of the NIR polaron band changes with dose, though not monotonically (Table 3). The initial dose causes a large $(23 \mathrm{~nm})$ blue shift, while subsequent doses gradually shift the peak back towards the near-infrared. There are not any corresponding shifts in the $445 \mathrm{~nm}$ band position. The width of this absorption band remains fairly constant with increasing dose.

The intensity of the polaron absorption bands generally decreases with dose, although it is hard to quantify the effect due to shifting baselines for the different samples and the possibility of varying thicknesses of the PANi layer between samples. The intensity trend roughly matches the observations of the Raman spectra, for which the PANi peaks were clearly diminished relative to PET peaks at $10^{8}$ rad and destroyed at $10^{9}$ rad.

Irradiation of PANi in $\mathrm{D}_{2}$ (Figure 8) generates similar types of changes to those observed for air-irradiated samples, although the magnitude of the change is reduced in each case. The growth of the "yellowing" UV band at the higher doses is not as strong. The polaron absorbance bands do not disappear completely, which is consistent with the persistence of the PANi Raman peaks for these samples. The initial red shift of the NIR band is not as great.

Table 3. Dose dependence of peak absorbance of PANi polaron band.

\begin{tabular}{|c|c|c|}
\hline Dose (rad) & $\boldsymbol{\lambda}_{\text {Max }}$ air $(\mathbf{n m})$ & $\boldsymbol{\lambda}_{\text {Max }}, \mathbf{D}_{\mathbf{2}}(\mathbf{n m})$ \\
\hline 0 & 792 & 792 \\
\hline $10^{5}$ & 763 & 775 \\
\hline $10^{6}$ & 763 & 770 \\
\hline $10^{7}$ & 774 & 793 \\
\hline $10^{8}$ & 803 & 797 \\
\hline $10^{9}$ & (no peak) & 803 \\
\hline
\end{tabular}

\section{Absorption spectroscopy of irradiated PEDOT-PSS samples}

The only observed absorption for the PEDOT-PSS samples is that of the PET substrate. The dose dependence for these samples matches that observed for the PANi samples with respect to the growth of the UV absorbance bands. Not only do the PEDOT-PSS air samples show greater UV absorbance for a given dose than the $\mathrm{D}_{2}$ samples, but the PEDOT-PSS air samples have the same UV absorbance as the PANi air samples (and likewise for the $\mathrm{D}_{2}$ samples). 


\section{Discussion of spectroscopy results}

The various observations for the Raman and absorbance spectra allow certain conclusions about the fate of the conducting PANi films when they are irradiated.

There is no observable absorbance that emerges at $590 \mathrm{~nm}$ for any dose, whether in air or $\mathrm{D}_{2}$. This implies that there is no significant conversion from the conductive emeraldine salt to the non-conductive emeraldine base form. Therefore the irradiation does not seem to affect the degree of protonation of the polymer. This conclusion is supported by the lack of any substantial changes of the PANi bands in the Raman spectra. The conclusion is also consistent with the analysis of the gas headspace after exposure to $T_{2}$, which indicated minimal hydrogen exchange with the environment. Therefore, conductivity changes are not due to an intact PANi changing its base form.

The most notable feature of both the Raman and absorbance spectra is the loss of intensity of bands associated with PANi for the $10^{8}$ and $10^{9}$ rad samples in air, but not in $\mathrm{D}_{2}$. This is generally consistent with the greater decreases in conductivity for the air samples and reinforces the role of an oxidative mechanism for the PANi degradation. No new Raman peaks, for example those which might be attributed to peroxy radicals, were observed for the doses at which the conductivity changed. However, several factors can explain the inability to see these peaks. The PET substrate has ester moieties with peaks ( $\sim 870$ and 1700 $1750 \mathrm{~cm}^{-1}$ ) that interfere with any scattering by the peroxy groups. The substrate is also much thicker than the conducting layer, so new bands from the conducting layer will be smaller than the existing bands. Any reacted polymer may lose its extended conjugation and not be resonantly enhanced by the $785 \mathrm{~nm}$ excitation, further reducing its Raman signal. Furthermore, the strength of the NIR absorbance band does not change substantially until the highest doses. Thus, only a small fraction of the PANi may undergo oxidative attack at the lower doses.

The observed intensity losses at the higher doses for both Raman and absorbance spectroscopy echo the results reported by Mažeikienè et al. [Mažeikienè , 2008]. These authors used in-situ Raman spectroscopy to monitor the effects of electrochemical treatment of a polyaniline film deposited on a gold electrode. Their excitation wavelength, $676 \mathrm{~nm}$, should allow observation of both the emeraldine salt and base structures. The Raman spectra showed only minor changes in peak shapes and distribution, and the most significant effect observed was a loss of overall intensity associated with degradation of the polymer layer. In both these experiments and those reported here, PANi was subjected to electronic attack (electrochemical or radiolysis-induced respectively). Bulk conversion into an observable, different form of the polyaniline seems less likely than formation of a completely different material through oxidation.

Interpretation of the wavelength shifts of the NIR absorption band is more problematic. The position of this peak and the overall shape of the band are determined by several effects. Extended conjugation length will tend to move the peak to lower energy (red shift). The physical conformation of the polymer and its relation to its environment may also affect the spectra. An example of the latter effect was observed by Yin and Rubinstein for doped PANi in solution [Yin, 2000]. Changes in the doping of the PANi led to shifts that were interpreted as being due to changes in the coiling of the polymer based on increased interaction energy with the solvent.

The similar trends of peak position shifts for the samples irradiated in air and $\mathrm{D}_{2}$ suggest that the observed behavior cannot be explained by an oxidative mechanism. Apart from the intensity losses for the airirradiated samples, the polaron band largely maintains its character throughout the doping process. The peak position undergoes moderate change, and the peak width remains essentially constant. These behaviors suggest that the explanation should involve a mild effect that does not significantly change the character of the PANi layer. One possible explanation could be an interaction of the PANi with the DNNSA acid dopant. The sulfonic acid $\left(-\mathrm{SO}_{2} \mathrm{OH}\right)$ moiety on DNNSA should undergo some interaction with $\gamma$ radiation, either 
yielding a radical that can interact with the PANi, or becoming ionized. If the irradiation process also allows the polymer a chance to anneal, the PANi could rearrange to a lower-energy configuration, which may or may not include enhanced cross-linking, which would be consistent with the observed red shift. Note that changes to the DNNSA would probably not be observed in the Raman spectrum, since it is present in small amounts and is not resonantly enhanced under our experimental conditions.

The PEDOT-PSS films do not have the NIR absorption bands that serve as an indication of conjugation length and provide a resonance enhancement for Raman measurements. Thus, it is not possible to make a full interpretation of the spectroscopic results as is done for the PANi films. However, the Raman results for the two films are similar. Any oxidative change to the PEDOT-PSS is not observable against the background ester bands of the PET substrate. Loss of band intensity at the higher doses in air, but not $\mathrm{D}_{2}$, indicates an oxidative mechanism for destruction of the films, though this is not accompanied by crazing or cracking of the conductive material.

\section{SUMMARY and CONCLUSIONS}

In summary, it has been shown that these proposed materials (PANi and PEDOT-PSS) both exhibit a measurable change in conductivity, even in low total doses of tritium gas, and have potential use as a tritium sensor. It is also important to note that varying the dose rate also provides a varied response in conductivity. Polyaniline provided a consistent decrease in measured conductivity with increase in dose, which would make calibration of a sensor easier and the response more predictable. However, PANi was shown through conductivity measurements and spectroscopy to be more susceptible to oxidation by air exposure. The oxidation problem may be solved easily with a radiation-transparent coating that is impermeable to oxygen.

Through spectroscopy, the mechanisms for polymer degradation by radiolysis and oxidation were elucidated, although further work would provide a more complete analysis of the mechanisms for conductivity change in the polymeric materials. Physical changes (e.g. C-C bond breakage), rather than chemical changes to the polymer were more evident based on the analyses made here.

\section{FUTURE WORK}

Future work for this program involves the design and fabrication of a prototype sensor device. This device will be fully tested with very small amounts of tritium gas (ppm level) exposed to the conducting polymer, more promisingly the polyaniline (PANi), to determine the overall sensitivity of this proposed device. In this way, the device can be fully calibrated. Additionally, a radiation-transparent, oxygen impermeable coating will be applied to the PANi surface if it is determined that the sensing device will be placed in an oxygen or air environment.

\section{REFERENCES}

Biskman, B. A. ; Klinshpont, E. R. ; Stepanov, V. F. ; Tlebaev, K. B. ; Journal of Spacecraft and Rockets 2004, vol. 41, 360-365

Chan, H.S.O.; Ng, S.C., Ho, P.K.H. Macromolecules (2002). 27: 2159-2164.

Clegg, D.W.; Collyer, A.A. Irradiation Effects on Polymers. Elsevier Applied Science. New York, NY, 1991.

Clough, R. L.; Shalaby, S.W., editors. Irradiation of Polymers. ACS Symposium Series 620. American Chemical Society, Washington, DC 1994.. 
Clough, R. L.; Shalaby, S.W. Irradiation of Polymers: Fundamentals and Technical Applications ACS Symposium Series 620. American Chemical Society, Washington, D.C., 1996.

de Acevedo, W. M.; da Costa Lima, A. P.; de Araujo, E. S. Radiation Protection Dosimetry 84 (1999) 77-81.

Garreau, S.; Louarn, G.; Buisson, J.P.; Froyer, G.; Lefrant, S. Macromolecules (1999) 32:6807-6812.

Gerard, M.; Chaubey, A.; Malhotra, B. D. Biosensors \& Bioelectronics 17 (2002) 345-359.

G. Inzelt; M. Pineri; J.W. Schultze; M.A. Vorotyntsev Electrochimica Acta 45 (2000) 2403-2421

Kim, J.-Y.; Lee, J.-H.; Kwon, S.-J. Synthetic Metals (2007) 336-342.

Lima Pachoco, A. P.; Araujo, E. S.; de Acevedo, W. M. Materials Characterization 50 (2003) 245-248.

Mažeikienè, R.; Niaura, G.; Malinauskas, A. Polymer Degradation and Stability (2008) 93:1742-1746.

Michielsen, S. Handbook of Raman Spectroscopy; Marcel Dekker: New York, 2001; Ch. 19.

Yin, W.; Ruckenstein, E. Synthetic Metals (2000) 108:39-46.

\section{ACKNOWLEDGEMENTS}

The authors wish to acknowledge Greg Staack and Dante Pilgrim for assistance with the tritium exposures, Gregg Creech and Chris Beam for assistance with the gamma exposures and Tommy Sessions for postexposure gas analysis. 